\title{
Adiabatic out-of-equilibrium solutions to the Boltzmann equation in warm inflation
}

\author{
Mar Bastero-Gil, ${ }^{a}$ Arjun Berera, ${ }^{b}$ Rudnei O. Ramos $^{c}$ and João G. Rosa ${ }^{d}$ \\ ${ }^{a}$ Departamento de Física Teórica y del Cosmos, Universidad de Granada, \\ Granada-18071, Spain \\ ${ }^{b}$ School of Physics and Astronomy, University of Edinburgh, \\ Edinburgh, EH9 3FD, United Kingdom \\ ${ }^{c}$ Departamento de Física Teórica, Universidade do Estado do Rio de Janeiro, \\ 20550-013 Rio de Janeiro, RJ, Brazil \\ ${ }^{d}$ Departamento de Física da Universidade de Aveiro and CIDMA, \\ Campus de Santiago, 3810-183 Aveiro, Portugal \\ E-mail: mbg@ugr.es, ab@ph.ed.ac.uk, rudnei@uerj.br, joao.rosa@ua.pt
}

ABSTRACT: We show that, in warm inflation, the nearly constant Hubble rate and temperature lead to an adiabatic evolution of the number density of particles interacting with the thermal bath, even if thermal equilibrium cannot be maintained. In this case, the number density is suppressed compared to the equilibrium value but the associated phasespace distribution retains approximately an equilibrium form, with a smaller amplitude and a slightly smaller effective temperature. As an application, we explicitly construct a baryogenesis mechanism during warm inflation based on the out-of-equilibrium decay of particles in such an adiabatically evolving state. We show that this generically leads to small baryon isocurvature perturbations, within the bounds set by the Planck satellite. These are correlated with the main adiabatic curvature perturbations but exhibit a distinct spectral index, which may constitute a smoking gun for baryogenesis during warm inflation. Finally, we discuss the prospects for other applications of adiabatically evolving out-of-equilibrium states.

Keywords: Cosmology of Theories beyond the SM, Thermal Field Theory

ArXiv EPrint: 1711.09023 


\section{Contents}

1 Introduction 1

2 Adiabatic out-of-equilibrium dynamics 2

3 Adiabatic baryogenesis during warm inflation 10

4 Generation of baryon isocurvature perturbations $\quad 12$

5 Summary and future prospects $\quad 15$

$\begin{array}{ll}\text { A Boltzmann equation for scattering processes } & 17\end{array}$

\section{Introduction}

Equilibrium and out-of-equilibrium dynamics play central roles in cosmology, being crucial in determining, for instance, the present abundances of light nuclei, dark matter and other thermal relics or the cosmological baryon asymmetry. From the nearly perfect blackbody spectrum of the Cosmic Microwave Background (CMB) radiation and the successful predictions of Big Bang Nucleosynthesis (BBN) for the abundances of Helium and other light nuclei, we can infer that the Universe achieved a state very close to thermal equilibrium in the early stages of its evolution. Most of the cosmological dynamics is based on determining when a given particle species was in equilibrium with the thermal cosmic plasma and when it decoupled from the latter. This generically involves solving intricate systems of coupled Boltzmann equations for different particle species, but it typically suffices to use the well-known "rule of thumb" that a particle is in equilibrium with the cosmic plasma whenever its interaction rate with the latter exceeds the Hubble expansion rate, $\Gamma \gtrsim H$. Since in most cosmological eras the Hubble rate varies significantly in a Hubble time, this implies that the transition from an equilibrium to an out-of-equilibrium (decoupled) state is very fast on cosmological time scales.

An exception to this rule is naturally the period of inflation [1-5], where the Hubble rate remains nearly constant for about 50-60 e-folds of expansion required to solve the flatness and horizon problems of the Big Bang model. In canonical models of inflation the question of whether or not a particle species is in thermal equilibrium makes little sense, since the exponentially fast expansion quickly dilutes away all particle species present in the pre-inflationary epochs. However, in the context of warm inflation $[6,7]$ this question plays a prominent role. In this alternative paradigm, dissipative processes continuously transfer the inflaton's energy into the cosmic plasma, leading to particle production that sustains a slowly varying temperature during inflation. There are several reasons to consider such an alternative paradigm. In such scenarios, dissipative friction damps the inflaton's evolution and prolongs inflation, with radiation smoothly taking over as the dominant component 
if strong dissipation is attained at the end of the slow-roll evolution regime (see, e.g., refs. [8-11]). The greatest appeal of warm inflation lies perhaps in the fact that thermal inflaton fluctuations are directly sourced by dissipative processes, changing the form of the primordial spectrum of curvature perturbations and thus providing a unique observational window into the particle physics behind inflation [11-18]. In addition, we have recently shown that warm inflation can be consistently realized in a simple quantum field theory framework requiring very few fields, the Warm Little Inflaton scenario [19], where the required flatness of the inflaton potential is not spoiled by thermal effects (see also refs. [20-26] for earlier alternative models), paving the way for developing a complete particle physics description of inflation that can be fully tested with CMB and Large-Scale Structure (LSS) observations and possibly have implications for collider and particle physics data.

Independently of the particle physics involved in sustaining a thermal bath during inflation, a generic feature of warm inflation is the slow evolution of both the temperature and the Hubble parameter for the usually required 50-60 e-folds of expansion. Since both scattering and particle decay rates typically depend on the former, this implies that the ratio $\Gamma / H$ will generically evolve slowly during inflation. Consequently, as we explicitly show in this work for the first time, particle species in the warm inflationary plasma can maintain distributions that are slowly evolving and out-of-equilibrium throughout inflation, whether or not they are directly involved in the dissipative dynamics. This may have an important impact not only on the inflationary dynamics and predictions themselves but also on the present abundance of different components. As an example of application of this novel observation, we show that this can lead to the production of a baryon asymmetry during inflation, a possibility that can be tested in the near future with CMB and LSS observations.

This work is organized as follows. In section 2 we analyze the Boltzmann equation for a particle species interacting with a thermal bath for an adiabatic evolution of the ratio $\Gamma / H$, focusing explicitly on the case of decays and inverse decays for concreteness. We then show that this leads to slowly varying out-of-equilibrium configurations, obtaining the overall particle number density and its phase space distribution. We briefly discuss how similar results can be obtained for scattering processes. In section 3 we use these results to develop a generic baryogenesis (or leptogenesis) mechanism during inflation. Then, in section 4, we discuss how this generically leads to baryon isocurvature modes that give a small contribution to the primordial curvature perturbation spectrum. In section 5 we summarize our results and discuss their potential impact on other aspects of the inflationary and post-inflationary history. An appendix is included where some technical details are given.

\section{Adiabatic out-of-equilibrium dynamics}

Let us consider a particle $X$ interacting with a thermal bath at temperature $T$ in an expanding Universe. ${ }^{1}$ For concreteness, let us explicitly consider the case where $X$ decays into particles $Y_{1}$ and $Y_{2}$ in the thermal bath, and assume that these maintain a near

\footnotetext{
${ }^{1}$ Throughout this work we consider a spatially flat Friedmann-Lemaitre-Robertson-Walker (FLRW) space-time in which the metric is given by $d s^{2}=d t^{2}-a(t)^{2} d \mathbf{x}^{2}$, where $t$ is physical time, $\mathbf{x}$ are the comoving spatial coordinates and $a(t)$ is the cosmological scale factor.
} 
equilibrium distribution through other processes that do not directly involve $X$. Let us also, for simplicity, focus on the case where $X$ is a boson with $g_{X}$ degrees of freedom and the decaying products $Y_{1}$ and $Y_{2}$ are either bosons or fermions (extending our results to decaying fermions is straightforward). The number density of $X$ particles,

$$
n_{X}=g_{X} \int \frac{d^{3} p}{(2 \pi)^{3}} f_{X}(\mathbf{p}),
$$

has an evolution, in an expanding flat FLRW universe, that is described by the Boltzmann equation $[27,28]$

$$
\dot{n}_{X}+3 H n_{X}=C,
$$

where the collision term $C$ includes the effects of decays $X \rightarrow Y_{1}+Y_{2}$ and inverse decays $Y_{1}+Y_{2} \rightarrow X$ and takes the form ${ }^{2}$

$C=-\int d \Pi_{X} d \Pi_{1} d \Pi_{2}(2 \pi)^{4} \delta^{4}\left(p_{X}-p_{1}-p_{2}\right)|\mathcal{M}|^{2}\left[f_{X}\left(1 \pm f_{1}^{\mathrm{eq}}\right)\left(1 \pm f_{2}^{\mathrm{eq}}\right)-f_{1}^{\mathrm{eq}} f_{2}^{\mathrm{eq}}\left(1+f_{X}\right)\right]$,

where $d \Pi_{i}=g_{i} d^{3} p_{i} / 2 E_{i}(2 \pi)^{3}, \mathcal{M}$ is the matrix element associated with both the decay and inverse decay processes (related by CPT invariance) and $f_{i}$ are the phase-space distribution functions for each particle. Note also that the plus (minus) sign in $1 \pm f_{i}$ in eq. (2.3) refers to bosons (fermions) and it is related to the usual Bose enhancement (Pauli-blocking) effect. Since we assume $Y_{1,2}$ are in equilibrium, we have

$$
f_{i}^{\mathrm{eq}}=\frac{1}{e^{\beta\left(E_{i}-\mu_{i}\right)} \pm 1}, \quad i=1,2
$$

where $\beta=1 / T$ in natural units and the plus (minus) sign is for a Fermi-Dirac (BoseEinstein) distribution. Taking into account conservation of energy, $E_{X}=E_{1}+E_{2}$, and assuming chemical equilibrium, $\mu_{X}=\mu_{1}+\mu_{2}$ (an assumption that we may drop if chemical potentials can be neglected), then, it is easy to show that

$$
1 \pm f_{1}^{\mathrm{eq}} \pm f_{2}^{\mathrm{eq}}=\frac{f_{1}^{\mathrm{eq}} f_{2}^{\mathrm{eq}}}{f_{X}^{\mathrm{eq}}}
$$

where

$$
f_{X}^{\mathrm{eq}}=\frac{1}{e^{\beta\left(E_{X}-\mu_{X}\right)}-1}
$$

is the distribution of the $X$ particles when they are in equilibrium. This then allows us to write the collision term in the form

$$
\begin{aligned}
C & =-\int d \Pi_{X} d \Pi_{1} d \Pi_{2}(2 \pi)^{4} \delta^{4}\left(p_{X}-p_{1}-p_{2}\right)|\mathcal{M}|^{2} \frac{f_{1}^{\mathrm{eq}} f_{2}^{\mathrm{eq}}}{f_{X}^{\mathrm{eq}}}\left(f_{X}-f_{X}^{\mathrm{eq}}\right) \\
& =-\int \frac{d^{3} p_{X}}{(2 \pi)^{3}} \Gamma_{X}\left(f_{X}-f_{X}^{\mathrm{eq}}\right)
\end{aligned}
$$

\footnotetext{
${ }^{2}$ The inclusion of Landau damping effects will not change our conclusions.
} 
where the equilibrium decay width of the $X$ boson is given by

$$
\Gamma_{X}=\frac{1}{2 E_{X} f_{X}^{\mathrm{eq}}} \int d \Pi_{1} d \Pi_{2}(2 \pi)^{4} \delta^{4}\left(p_{X}-p_{1}-p_{2}\right)|\mathcal{M}|^{2} f_{1}^{\mathrm{eq}} f_{2}^{\mathrm{eq}} .
$$

We can simplify further the Boltzmann equation by discarding the momentum-dependence of the decay width, a common procedure in the literature, by considering its thermal average [27]:

$$
\bar{\Gamma}_{X}=\frac{1}{n_{X}^{\mathrm{eq}}} \int d^{3} p_{X} \Gamma_{X} f_{X}^{\mathrm{eq}}
$$

The Boltzmann equation (2.2) can then be cast into the familiar form

$$
\dot{n}_{X}+3 H n_{X}=-\bar{\Gamma}_{X}\left(n_{X}-n_{X}^{\mathrm{eq}}\right) .
$$

It is straightforward to obtain an analogous result for an arbitrary number of particles in the final state.

In a non-expanding Universe, the collision term will then naturally drive the number density of $X$ particles towards its equilibrium value, while in an expanding Universe this only occurs for $\bar{\Gamma}_{X} \gg H$, which is the familiar rule of thumb in cosmology. Now, in a cosmological setting such as warm inflation, since $H$ and $T$ are slowly-varying and $\bar{\Gamma}_{X}$ will depend only on $T$ and on the masses of the parent and daughter particles, we can take the ratio $\bar{\Gamma}_{X} / H$ to be slowly-evolving. For all cases where $\gamma_{X} \equiv \bar{\Gamma}_{X} / H$ is slowly-evolving on the Hubble scale, i.e., $\dot{\gamma}_{X} / \gamma_{X} \ll H^{-1}$, we may take $\gamma_{X}$ to be a constant as a first approximation and write the Boltzmann equation in terms of the number of e-folds of expansion, $d N_{e}=H d t$, as

$$
n_{X}^{\prime}+3 n_{X}=-\gamma_{X}\left(n_{X}-n_{X}^{\mathrm{eq}}\right)
$$

If the temperature is slowly-varying, we may take $n_{X}^{\mathrm{eq}}$ to be constant as well, yielding the solution

$$
n_{X}\left(N_{e}\right)=\frac{\gamma_{X}}{3+\gamma_{X}} n_{X}^{\mathrm{eq}}\left(1-e^{-\left(3+\gamma_{X}\right) N_{e}}\right)+n_{X}(0) e^{-\left(3+\gamma_{X}\right) N_{e}} .
$$

Hence, $X$ is driven exponentially fast to the solution

$$
n_{X} \simeq \frac{\gamma_{X}}{3+\gamma_{X}} n_{X}^{\mathrm{eq}}
$$

Note that this is not exactly stationary due to the slow variation of both $\gamma_{X}$ and $n_{X}^{\mathrm{eq}}$, so we refer to this solution as adiabatic. This solution is attained in less than a e-fold if the initial number density is not too far from the quasi-stationary value, and even large discrepancies will be quickly washed away within a few e-folds. This agrees with the statement made above that $n_{X}$ is driven towards its equilibrium value for $\gamma_{X} \gg 1$, but reveals a novel feature that is absent in general cosmological settings - that even for $\gamma_{X} \ll 1$ a small but not necessarily negligible number density of $X$ particles remains constant despite the fast expansion of the Universe. This is thus a new type of solution that only arises in cosmological 
settings where the temperature and Hubble rate are varying slowly, such as warm inflation. In an adiabatic approximation, we can include the slow variation of these parameters, namely $\gamma_{X}=\gamma_{X}\left(N_{e}\right)$ and $n_{X}^{\mathrm{eq}}=n_{X}^{\mathrm{eq}}\left(N_{e}\right)$ in the quasi-stationary solution in eq. (2.12).

We may also take a step further and compute the phase-space distribution of $X$ particles, which follows the momentum-dependent Boltzmann equation in a flat FLRW universe,

$$
\frac{\partial f_{X}(\mathbf{p}, t)}{\partial t}-H p \frac{\partial f_{X}(\mathbf{p}, t)}{\partial p}=C(\mathbf{p})
$$

where the collision term is given by

$$
C(\mathbf{p})=-\Gamma_{X}(\mathbf{p})\left[f_{X}(\mathbf{p}, t)-f_{X}^{e q}(\mathbf{p})\right],
$$

from the results obtained above. For illustrative purposes, we will consider two distinct cases where an $X$ scalar boson decays into either fermion or scalar boson pairs, through Yukawa or scalar trilinear interactions, respectively. We neglect all chemical potentials for simplicity. The corresponding decay widths are given by [23]

$$
\begin{aligned}
& \Gamma_{X}^{(B)}=\Gamma_{0}^{(B)} \frac{m_{X}}{\omega_{p}}\left[1+2 \frac{T}{p} \log \left(\frac{1-e^{-\omega_{+} / T}}{1-e^{-\omega_{-} / T}}\right)\right] \\
& \Gamma_{X}^{(F)}=\Gamma_{0}^{(F)} \frac{m_{X}}{\omega_{p}}\left[1+2 \frac{T}{p} \log \left(\frac{1+e^{-\omega_{+} / T}}{1+e^{-\omega_{-} / T}}\right)\right]
\end{aligned}
$$

where we have neglected the masses of the decay products, with $\omega_{ \pm}=\left(\omega_{p} \pm p\right) / 2$, $\omega_{p}=\sqrt{p^{2}+m_{X}^{2}}, p=|\mathbf{p}|$. The decay widths at zero-temperature and zero-momentum are, in the two cases, given by

$$
\Gamma_{0}^{(B)}=\frac{g_{B}^{2}}{32 \pi} \frac{M^{2}}{m_{X}}, \quad \Gamma_{0}^{(F)}=\frac{g_{F}^{2}}{8 \pi} m_{X}
$$

where $g_{B, F}$ are dimensionless couplings and $M$ is the mass scale of the trilinear scalar coupling. We have solved the Boltzmann equation (2.13) numerically in both cases for different values of the $X$ boson mass and decay width, taking $f_{X}(\mathbf{p}, 0)=0$ and imposing $f_{X}(\mathbf{p}, t) \rightarrow 0$ in the limit $p \rightarrow \infty$ (in practice at a sufficiently large momentum value). We illustrate the resulting time evolution of the phase-space distribution for the bosonic and fermionic cases in figure 1(a) and figure 1(b) respectively. In both cases the decay is of a relativistic $X$ boson with mass set at the value $m_{X}=0.001 T$ and with $\Gamma_{0}^{(B, F)} / H=0.5$, which corresponds to $\gamma_{X}^{(B)}=0.0245\left(\gamma_{X}^{(F)}=10^{-4}\right.$ in the case of decay into fermions). It is clear from the results shown in figure 1 that in both cases the distribution reaches a stationary configuration after only a couple of Hubble times, a result that we obtained generically for different values of the mass and decay width, for both fermionic and bosonic decay. Naturally, for larger values of $\gamma_{X}$ the stationary configuration is attained faster.

These stationary solutions can be obtained by setting $\partial f_{X} / \partial t=0$ in the Boltzmann equation (2.13), yielding a first-order inhomogeneous differential equation for $f_{X}^{\text {stat }}(\mathbf{p})$. Following standard methods, we may formally write the stationary solution in the integral form

$$
f_{X}^{\text {stat }}(p)=f_{X}^{(h)}(p) \int_{p}^{\infty} \frac{d p^{\prime}}{p^{\prime}} \frac{\Gamma_{X}\left(p^{\prime}\right)}{H} \frac{f_{X}^{\mathrm{eq}}\left(p^{\prime}\right)}{f_{X}^{(h)}\left(p^{\prime}\right)},
$$




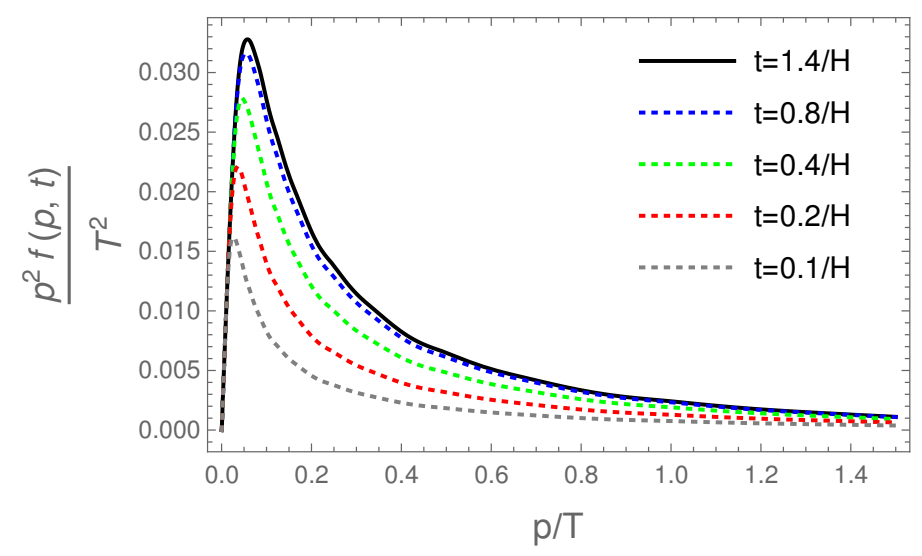

(a) Decay into bosons.

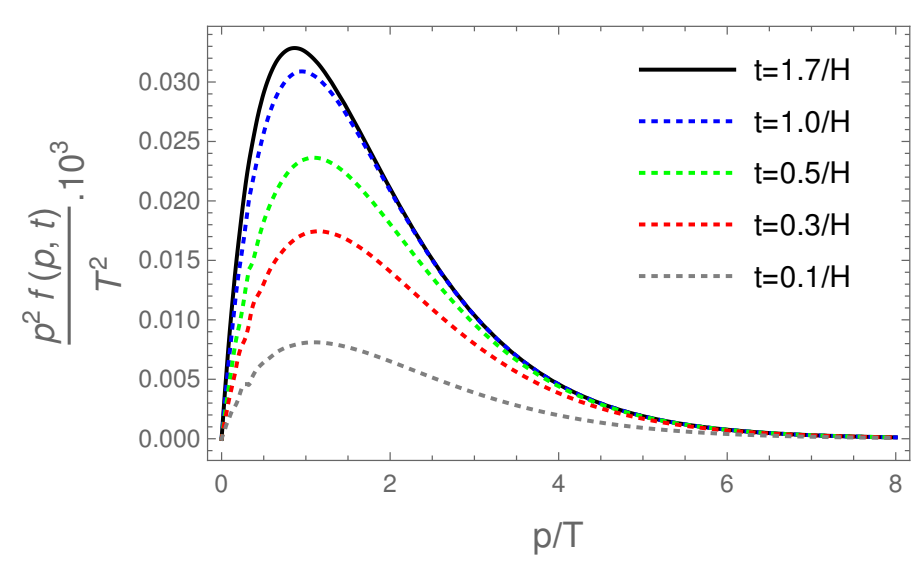

(b) Decay into fermions.

Figure 1. The phase space distribution at different times (in units of the Hubble parameter), as a function of the momentum $p$, for decay into bosons (a) and for decay into fermions (b). The parameters used are $m_{X}=0.001 T$, and $\Gamma_{0}^{(B, F)} / H=0.5$, which corresponds to $\gamma_{X}^{(B)}=0.0245$ (bosons) and $\gamma_{X}^{(F)}=10^{-4}$ (fermions).

where $f_{X}^{(h)}=e^{\int d p^{\prime} \Gamma\left(p^{\prime}\right) / H p^{\prime}}$ is the homogeneous solution, which takes the form $f_{X}^{(h)}=p^{\gamma_{X}}$ if one replaces the decay width by its thermal average eq. (2.8), as explained above.

For practical purposes, however, this integral form is not very useful, since integrals involving the Bose-Einstein distribution do not have, in general, a simple analytical form and have to be computed numerically. In figure 2 , we show the obtained stationary distributions for bosonic and fermionic decays for the same values of the $X$ mass and average decay width considered in figure 1 .

For comparison, we also show in figure 2 the distribution obtained when replacing $\Gamma_{X}(p)$ by $\bar{\Gamma}_{X}$. We can see that for fermionic decay this yields a good approximation to the full solution, while for bosonic decay there are more prominent differences. In particular, the latter distribution is peaked at lower momentum values than the one obtained using $\bar{\Gamma}_{X}$, which is related to the Bose enhancement of the decay at low-momentum values $p \lesssim m_{X}$. For $m_{X} \ll T$ there is thus a substantial variation of the bosonic decay width with 


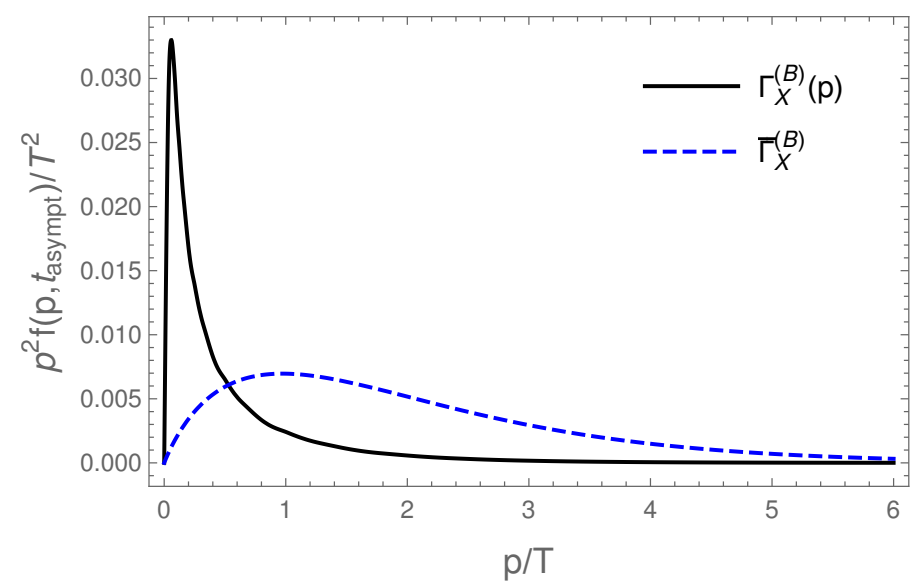

(a) Decay into bosons.

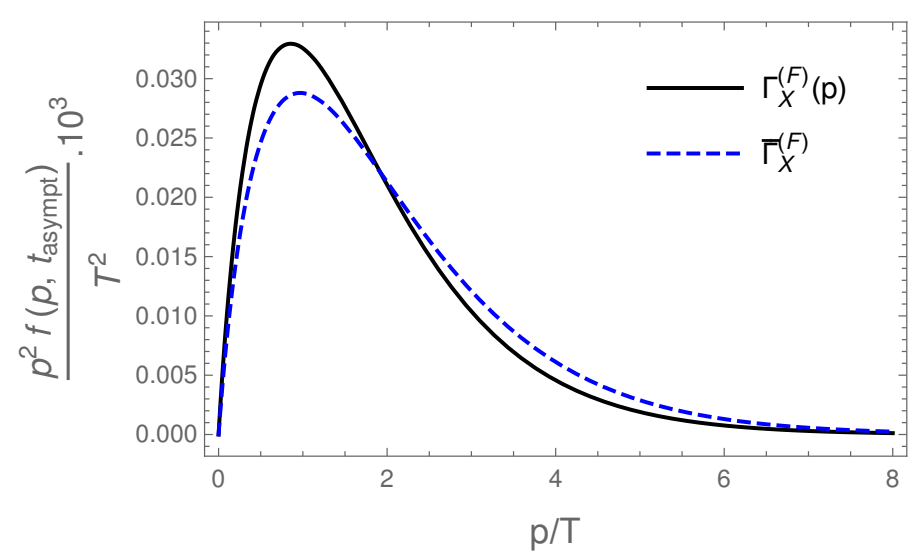

(b) Decay into fermions.

Figure 2. The phase space distribution, as a function of the momentum $p$, at large asymptotic times (when the distributions have already reached the stationary state), for decay into bosons (a) and for decay into fermions (b). The parameters used are $m_{X}=0.001 T$ and $\Gamma_{0}^{(B, F)} / H=0.5$, which corresponds to $\gamma_{X}^{(B)}=0.0245$ (bosons) and $\gamma_{X}^{(F)}=10^{-4}$ (fermions). Solid lines yield the solution when considering the full momentum dependent decay widths, while dashed lines correspond to the solution obtained using the constant thermally averaged decay widths.

momentum in the relevant range $p \lesssim T$, while for fermionic decay it is a good approximation to use the thermally averaged decay width in place of the full momentum-dependent expression. Note that the larger the mass of the $X$ boson the closer the distributions are to the one obtained using $\bar{\Gamma}_{X}$, since thermal corrections to the decay width become less important in this regime for $p \lesssim T \lesssim m_{X}$. All stationary distributions are nevertheless reasonably well fitted by an expression of the form

$$
f_{X}^{\text {stat }}(p)=A \frac{\Gamma_{X}\left(p, T_{\text {stat }}\right)}{3 H+\Gamma_{X}\left(p, T_{\text {stat }}\right)} \frac{1}{e^{\sqrt{p^{2}+m_{X}^{2}} / T_{\text {stat }}}-1} .
$$

We show in figure 3 the results for the fit amplitude $A$ and effective temperature $T_{\text {stat }}$ for $m_{X}=0.001 T$ and different values of the thermally averaged decay width, for both the bosonic and fermionic decays. 


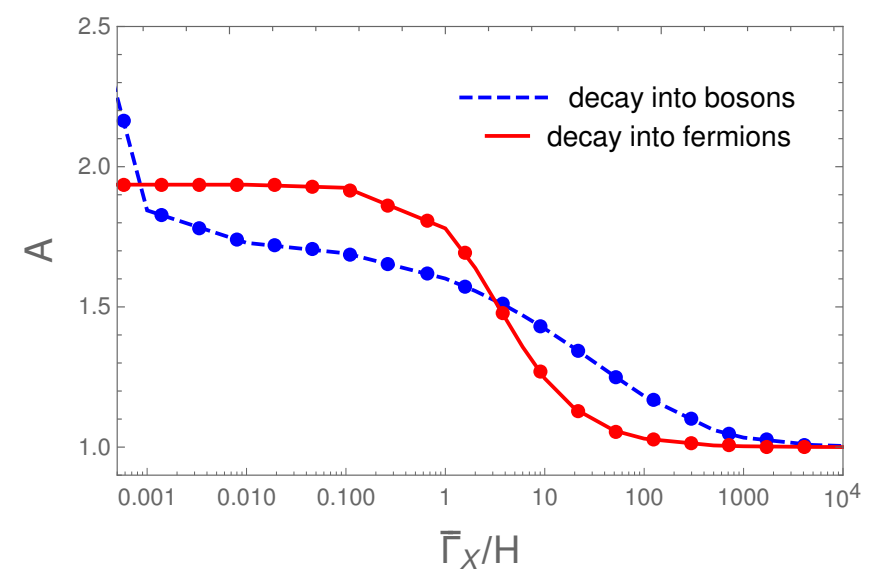

(a) The fit amplitude $A$.

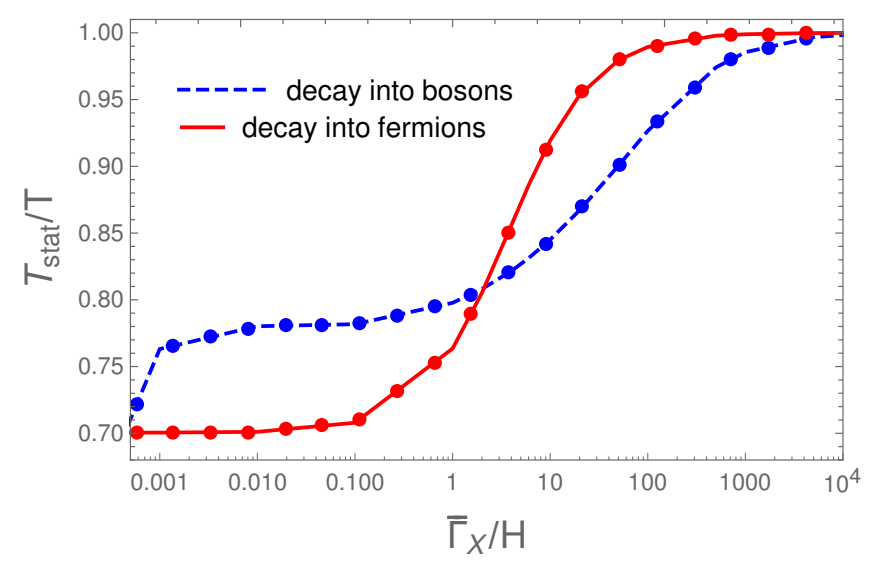

(b) The effective temperature $T_{\text {stat }}$.

Figure 3. The fitting amplitude $A$ (a) and effective temperature $T_{\text {stat }}$ (b) for the stationary distribution with $m_{X}=0.001 T$. Dashed lines are for the case of decay into bosons, while solid lines are for the case of decay into fermions.

As we can see in figure 3 , for relativistic $X$ bosons the coefficient $A \sim \mathcal{O}(1)$ for fermionic decays and also bosonic decays, unless the decay width is much smaller than the Hubble parameter, while the effective temperature $T_{\text {stat }} \lesssim T$. The larger variation of the fit parameters for bosonic decay is again due to the above mentioned Bose enhancement effect, a variation that becomes smaller for larger values of the $X$ mass. With the results shown in figure 3 , we also note that, generically, the effective temperature $T_{\text {stat }}$ approaches (asymptotically) the thermal bath temperature $T$ from below and likewise for the overall amplitude in front of eq. (2.18). This then means that the stationary solution $f_{X}^{\text {stat }}$ gets suppressed at large momenta relative to the equilibrium one $f^{\text {eq }}$. This result is similar to the one obtained recently for an exact solution of the Boltzmann equation in a FLRW background $[29,30]$, though it differs fundamentally from the solution found here. In particular, the result of refs. [29, 30] applies to a massless gas of particles with MaxwellBoltzmann distribution in a radiation dominated epoch and it only approaches a stationary 


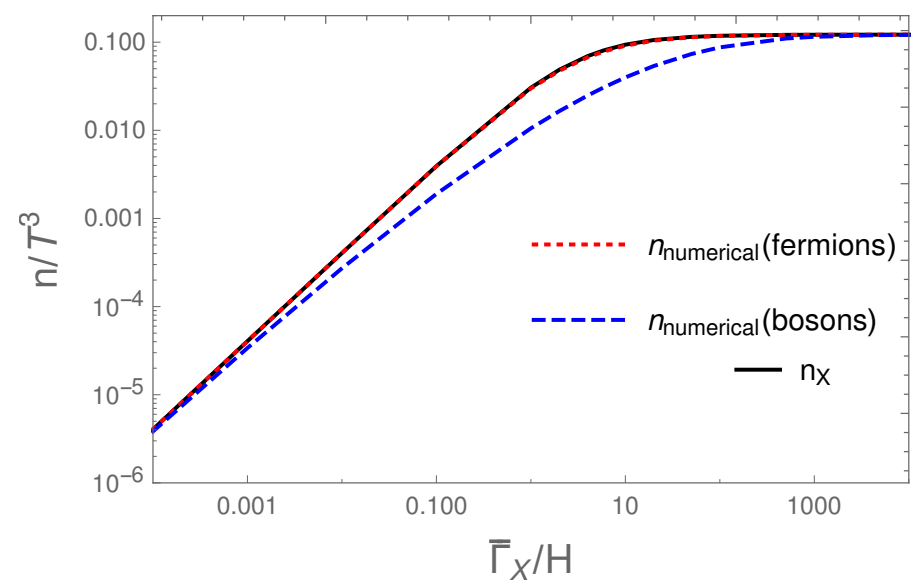

Figure 4. The momentum integrated distributions for the cases of decay into bosons (dashed line) and decay into fermions (dotted line). The solid line is the result of the solution given by eq. (2.12).

distribution at asymptotically large times, while the present one applies generically to a relativistic distribution in an inflationary regime and reaches a stationary state in only a few e-folds.

Finally, we can obtain explicitly the number density from our results. We have integrated the numerically obtained distributions over momenta and compared the results with the adiabatic solution for the number density in eq. (2.12). These results are shown in figure 4.

We conclude that eq. (2.12) is a good approximation to the numerical value of the number density, particularly for fermionic decays, while again we observe some discrepancies in the case of bosonic decays for intermediate values of $\bar{\Gamma}_{X} / H$. These only occur for relativistic $X$ bosons and eq. (2.12) becomes a better approximation as $m_{X}$ increases. We note that, in any case, the observed discrepancies correspond at most to $\mathcal{O}(1)$ factors, so that $\gamma_{X} /\left(3+\gamma_{X}\right) n_{X}^{\text {eq }}$ is generically a good approximation to the number density in the adiabatic evolution regime.

Our generic analysis would not be complete without considering scattering processes, which may also contribute to the collision term in the Boltzmann equation. Although, for the same type of interactions, scattering processes are typically suppressed compared to decays since they involve larger powers of the couplings in the perturbative regime, there may be cosmological settings where they correspond to different types of interactions and may dominate the collision term. The analysis of scattering processes is somewhat more involved than for decays, since in general one cannot easily express the collision term in terms of the number density, as we detail in appendix A. This is nevertheless possible in the limit of small occupation numbers, $f_{i} \ll 1$, i.e., in the absence of Bose enhancement or Fermi degeneracy [27]. In this regime, the Boltzmann equation can be written as

$$
\dot{n}_{X}+3 H n_{X} \simeq-\langle\sigma v\rangle\left(n_{X}^{2}-n_{X}^{\mathrm{eq} 2}\right),
$$

where $\langle\sigma v\rangle$ is the thermally averaged cross section times velocity defined in appendix A. In the adiabatic limit, where both the latter and $H$ vary slowly, we obtain the adiabatic 
solution for the number density

$$
n_{X} \simeq \frac{3}{2 \gamma_{X}^{(s)}}\left(\sqrt{1+\frac{4}{9} \gamma_{X}^{(s) 2}}-1\right) n_{X}^{\mathrm{eq}},
$$

where $\gamma_{X}^{(s)}=\langle\sigma v\rangle n_{X}^{\mathrm{eq}} / H$ is the ratio between the thermally averaged scattering rate and the Hubble parameter. Although this may seem more complicated than the adiabatic solution for decays, we note that the suppression factor with respect to the equilibrium number density becomes $\gamma_{X}^{(s)} / 3$ for small values of $\gamma_{X}^{(s)}$ and also tends to 1 in the limit $\gamma_{X} \gg 1$, as in eq. (2.12). The cases where the collision term is dominated by decays or by scattering processes thus yield quite similar adiabatic solutions for the number density, at least for small occupation numbers.

\section{Adiabatic baryogenesis during warm inflation}

The fact that we have obtained new solutions to the Boltzmann equation that are inherently out-of-equilibrium immediately suggests an application: the production of a baryon asymmetry during inflation. Let us then consider a generic model where the $X$ particles (bosons or fermions) decay violating $B$ (or $L$ or $B-L$ ) and $\mathrm{C} / \mathrm{CP}$. In particular, let us consider a simple case with two possible decay channels (as discussed e.g. in ref. [31]),

$$
X \rightarrow B, \quad X \rightarrow Y,
$$

where the final state $B$ carries a baryon number $b>0$ and $Y$ has no baryonic charge. In practical applications these will typically correspond to 2-body decays, although the number of particles in the final state can be arbitrary. $B$-violation then occurs because there is no consistent assignment of a baryonic charge to the $X$ particle. We also have the conjugate decays $\bar{X} \rightarrow \bar{B}$ and $\bar{X} \rightarrow \bar{Y}$ with opposite baryonic charges, and C/CP violation implies that the partial decay widths satisfy the relations

$$
\begin{aligned}
\Gamma(X \rightarrow B) & =\frac{1}{2}(1+\epsilon) \Gamma_{X}, \quad \Gamma(X \rightarrow Y)=\frac{1}{2}(1-\epsilon) \Gamma_{X}, \\
\Gamma(\bar{X} \rightarrow \bar{B}) & =\frac{1}{2}(1+\bar{\epsilon}) \Gamma_{X}, \quad \Gamma(\bar{X} \rightarrow \bar{Y})=\frac{1}{2}(1-\bar{\epsilon}) \Gamma_{X},
\end{aligned}
$$

where $\epsilon \neq \bar{\epsilon}$ yield the amount of $\mathrm{C} / \mathrm{CP}$ violation. Note that the total decay widths of the $X$ particle and of its anti-particle are equal, as required by CPT invariance,

$$
\Gamma(X \rightarrow B)+\Gamma(X \rightarrow Y)=\Gamma(\bar{X} \rightarrow \bar{B})+\Gamma(\bar{X} \rightarrow \bar{Y}) .
$$

Both $X$ and $\bar{X}$ will then obey the same Boltzmann equation (2.10) when the particles in the $B, Y$ final states are in equilibrium (which we assume to be maintained by other interactions), and evolve towards the adiabatic solution (2.12), which will be the same for both $n_{X}$ and $n_{\bar{X}}$. The full adiabatic solution (2.11) may be different for both $n_{X}$ and $n_{\bar{X}}$ if the initial values are distinct, but any discrepancies are quickly erased by expansion. 
Now, the baryon number density, i.e., the difference between the number density of baryons and that of anti-baryons, evolves according to the Boltzmann equation given by

$$
n_{B}^{\prime}+3 n_{B}=b\left[\frac{\gamma_{X}}{2}(1+\epsilon)\left(n_{X}-n_{X}^{\mathrm{eq}}\right)-\frac{\gamma_{X}}{2}(1+\bar{\epsilon})\left(n_{\bar{X}}-n_{\bar{X}}^{\mathrm{eq}}\right)\right] .
$$

Let us consider the case where $n_{X}(0)=n_{\bar{X}}(0)$, such that

$$
n_{B}^{\prime}+3 n_{B}=-b \frac{\gamma_{X}}{2} \Delta \epsilon\left(n_{X}-n_{X}^{\mathrm{eq}}\right)
$$

where $\Delta \epsilon=\bar{\epsilon}-\epsilon$ corresponds to the amount of $\mathrm{CP}$ violation. Using the solution given by eq. (2.11), this yields the adiabatic solution for the baryon number density,

$$
n_{B}\left(N_{e}\right) \simeq \frac{b}{2} \Delta \epsilon\left[\left(1-e^{-\left(3+\gamma_{X}\right) N_{e}}\right) \frac{\gamma_{X}}{3+\gamma_{X}} n_{X}^{\mathrm{eq}}+n_{X}(0) e^{-3 N_{e}}\left(e^{-\gamma_{X} N_{e}}-1\right)\right],
$$

which approaches (exponentially fast) the quasi-stationary solution,

$$
n_{B} \simeq \frac{b}{2} \Delta \epsilon \frac{\gamma_{X}}{3+\gamma_{X}} n_{X}^{\mathrm{eq}}
$$

Hence, we see that a constant baryon asymmetry is produced during warm inflation (or in fact during any analogous period of quasi-adiabatic temperature and Hubble rate evolution), for any value of $\gamma_{X}=\bar{\Gamma}_{X} / H$. Interestingly, $n_{B} \rightarrow b \Delta \epsilon n_{X}^{\text {eq }} / 2$ for $\gamma_{X} \rightarrow \infty$, the limit for which the $X$ particles are in equilibrium. This may seem to contradict Sakharov's conditions for baryogenesis [32], but it is simply associated with the fact that we are taking decays and inverse decays as the main processes responsible for driving the $X$ particles towards equilibrium. In this case, to get closer to equilibrium, we need to increase $\gamma_{X}$, which also increases the rate of production of baryon number, in such a way that we obtain a finite baryon number density for arbitrarily large $\gamma_{X}$. Note that, in the large $\gamma_{X}$ limit, from the solution given by eq. (2.11), we have that $n_{X}-n_{X}^{\mathrm{eq}} \rightarrow-3 n_{X}^{\mathrm{eq}} / \gamma_{X}$. Thus, the baryon source term on the right-hand-side of eq. (3.5) tends to a finite value $3 b \Delta \epsilon n_{X}^{\mathrm{eq}} / 2$ in the limit $\gamma_{X} \rightarrow+\infty$. However, note that in any physical setting $\gamma_{X}$ is not at the limiting value but rather is finite, which means the $X$ particles are always out-of-equilibrium. Nevertheless this analysis demonstrates that this parameter can be arbitrarily large and still produce a significant baryon asymmetry.

The baryon asymmetry produced during warm inflation can set the final cosmological asymmetry if the source term in the baryon number density equation becomes suppressed after the slow-roll regime and throughout the subsequent the cosmic history. This is, of course, model-dependent, but we can envisage scenarios where some other processes, such as e.g. scatterings, are more suppressed than decays during warm inflation, but become the dominant processes once the slow-roll period is over and radiation becomes the dominant component. In this case $X$ will be driven towards equilibrium after inflation more quickly than through decays and inverse decays and the baryon source will quickly shut down. If it remains in equilibrium until it is sufficiently non-relativistic, there should be no significant sources of baryon number at late times that could substantially modify the asymmetry produced during inflation. ${ }^{3}$

\footnotetext{
${ }^{3}$ Potentially electroweak sphalerons [33] may convert a lepton asymmetry into a baryon asymmetry in a leptogenesis scenario [34].
} 
The smallness of the observed cosmological baryon-to-entropy ratio $n_{B} / s$ can have different sources in this scenario: (i) CP-violation may be small, $\Delta \epsilon \ll 1$, (ii) the $X$ particles may be far from equilibrium, $\gamma_{X} \ll 1$ or (iii) $X$ particles may be non-relativistic during warm inflation, $n_{X}^{\mathrm{eq}} / s \ll 1$. Any combination of these may thus easily explain why $n_{B} / s \sim 10^{-10}$ (see, e.g., ref. [35] for BBN constraints on this ratio).

Let us consider in more detail the particular case where the $X$ particles are relativistic during inflation, $m_{X} \ll T$, as well as fully out-of-equilibrium, $\gamma_{X} \ll 1$. In this case, the baryon-to-entropy ratio is given by

$$
\frac{n_{B}}{s} \simeq \frac{45 \zeta(3)}{4 \pi^{4}} b \Delta \epsilon \frac{g_{X}}{g_{*}} \frac{\bar{\Gamma}_{X}}{H},
$$

where $g_{X}$ is the number of degrees of freedom in $X$, to which each bosonic (fermionic) degree of freedom contributes by a factor $1(3 / 4)$, and $g_{*}$ is the effective total number of relativistic degrees of freedom in the thermal bath. The smallness of the observed baryon-to-entropy ratio may in this case be due to a small amount of $\mathrm{CP}$ violation in $\Delta \epsilon$ and/or large deviations from thermal equilibrium, $\gamma_{X} \ll 1$, during inflation, or a combination of both these factors, with an additional suppression by $g_{X} / g_{*}$. This will of course be a model-dependent issue that is not pursued further here. We note that the high temperatures typically attained during warm inflation (see, e.g., refs. [17, 19]) suggest possible implementations of this mechanism within grand unified theories [36].

\section{Generation of baryon isocurvature perturbations}

The interesting aspect that we would like to discuss in more detail is the fact that $n_{B} / s$ depends on the ratio $\bar{\Gamma}_{X} / H$, which, albeit nearly constant, exhibits a small variation during inflation and, moreover, is associated with the inflationary dynamics. As such, this ratio will necessarily acquire fluctuations on superhorizon scales due to fluctuations in the inflaton field. This implies that the latter will induce both adiabatic curvature fluctuations and baryon isocurvature fluctuations, the latter corresponding to relative fluctuations in the baryon and photon fluids, which would be absent if the baryon asymmetry were not generated during inflation. This is similar to the warm baryogenesis scenario proposed in ref. [37], where the baryon asymmetry is directly sourced by the dissipative processes that sustain the thermal bath in warm inflation, but the spectrum of isocurvature modes may be different. This yields the interesting possibility of looking for baryon isocurvature modes with CMB and LSS observations to assess whether the observed baryon asymmetry was or not produced during inflation, but also whether its generation was directly linked with dissipative dynamics. Note that other baryogenesis models [38-43], such as Affleck-Dine baryogenesis, may also lead to baryon isocurvature modes in the primordial spectrum, but these are in this case uncorrelated with the main adiabatic curvature component, since they are associated with distinct fields. The degree of correlation between isocurvature and adiabatic curvature modes may thus be used to test the warm inflation paradigm itself and isolate a particular mechanism for baryogenesis.

Let us then analyze in more detail the spectrum of baryon isocurvature perturbations produced in the present model. Since $n_{B} / s$ will always depend on $\gamma_{X}$ in the adiabatic 
dynamics under consideration, baryon isocurvature modes will always be generated, independently of the value of $\gamma_{X}$ or whether $X$ particles are relativistic during inflation, but let us focus, for concreteness, on the weakly-coupled high-temperature case leading to eq. (3.8). At high temperature, we typically have that $\bar{\Gamma}_{X} \propto T$, such that $n_{B} / s \propto T / H$. Baryon isocurvature modes are characterized by the quantity [44]

$$
S_{B}=\frac{\delta \rho_{B}}{\rho_{B}}-\frac{3}{4} \frac{\delta \rho_{R}}{\rho_{R}}=\frac{\delta\left(n_{B} / s\right)}{n_{B} / s}=\frac{\delta(T / H)}{T / H}
$$

evaluated when the relevant CMB scales become superhorizon during inflation. The subscript ' $R$ ' used in eq. (4.1) and in the quantities below refers to radiation. We thus need to determine how the fluctuations in the ratio $T / H$ are related to inflaton fluctuations. The dynamics of warm inflation is dictated by the coupled inflaton and radiation equations, which for the homogeneous background components are given by

$$
\begin{aligned}
\ddot{\phi}+(3 H+\Upsilon) \dot{\phi}+V^{\prime}(\phi) & =0, \\
\dot{\rho}_{R}+4 H \rho_{R} & =\Upsilon \dot{\phi}^{2}
\end{aligned}
$$

where $\Upsilon$ is the dissipation coefficient. In the slow-roll regime, valid when the slow-roll parameters $\epsilon_{\phi},\left|\eta_{\phi}\right| \ll 1+Q$, where $Q=\Upsilon / 3 H, \epsilon_{\phi}=\left(M_{P}^{2} / 2\right)\left(V^{\prime} / V\right)^{2}$ and $\eta_{\phi}=M_{P}^{2} V^{\prime \prime} / V$, these become

$$
\dot{\phi} \simeq-\frac{V^{\prime}(\phi)}{3 H(1+Q)}, \quad \rho_{R} \simeq \frac{3}{4} Q \dot{\phi}^{2} .
$$

Combining both equations for $\rho_{R}=C_{R} T^{4}$, where $C_{R}=\left(\pi^{2} / 30\right) g_{*}$, we obtain after some algebra, that

$$
\left(\frac{T}{H}\right)^{4} \simeq \frac{3}{2} C_{R}^{-1}\left(\frac{M_{P}}{H}\right)^{2} \frac{Q}{(1+Q)^{2}} \epsilon_{\phi}
$$

where one can see that a warm thermal bath, i.e., $T \gtrsim H$, can easily be attained for $H \ll M_{P}$ in the slow-roll regime even if the dissipative ratio $Q$ is not very large. Considering perturbations in the above equation, we obtain after a straightforward calculation,

$$
S_{B}=\frac{\delta(T / H)}{T / H} \simeq \frac{1}{4}\left(\frac{6 \epsilon_{\phi}-2 \eta_{\phi}}{1+Q}+\frac{1-Q}{1+Q} \frac{Q^{\prime}}{Q}\right) \mathcal{R}
$$

where $\mathcal{R} \simeq(H / \dot{\phi}) \delta \phi$ is the gauge-invariant comoving curvature perturbation (written in the $\Psi=0$ gauge [28]) and primes denote derivatives with respect to the number of efolds of inflation, $d N_{e}=H d t$. The dynamical quantity $Q^{\prime} / Q$ depends on the form of the dissipation coefficient $\Upsilon$, but it is in general a linear combination of the slow-roll parameters divided by a linear polynomial in $Q$ (see, e.g., refs. $[9,17,19]$ ). This implies that typically $S_{B} / \mathcal{R} \sim \mathcal{O}\left(n_{s}-1\right) \sim \mathcal{O}\left(N_{e}^{-1}\right)$.

The Warm Little Inflaton (WLI) scenario of ref. [19], where the inflaton interacts with relativistic fermion fields and $\Upsilon \propto T$, constitutes the simplest and most appealing particle physics realization of warm inflation. In this case, we have $Q^{\prime} / Q=\left(6 \epsilon-2 \eta_{\phi}\right) /(3+5 Q)$. 
For thermal inflaton fluctuations and weak dissipation at horizon-crossing, $Q_{*} \ll 1$, we have for the scalar spectral index $n_{s}-1=2\left(2 \eta_{\phi_{*}}-6 \epsilon_{\phi_{*}}\right) / 3$, yielding

$$
S_{B}^{\mathrm{WLI}} \simeq\left(\frac{1-n_{s}}{2}\right) \mathcal{R} \simeq 10^{-2} \mathcal{R}
$$

Although the exact relation between $S_{B}$ and $\mathcal{R}$ is model-dependent, we expect these quantities to be in general proportional with a proportionality constant of this magnitude as argued above. We note that the effects of baryon and cold dark matter isocurvature modes (CDI) on the CMB spectrum are indistinguishable, although, e.g., the trispectrum may in principle distinguish between them [45]. As such, the effective contribution to the cold dark matter isocurvature spectrum from the baryon modes above is given by

$$
P_{\mathrm{CDI}}=\left(\frac{\Omega_{B}}{\Omega_{c}}\right)^{2}\left(\frac{S_{B}}{\mathcal{R}}\right)^{2} P_{\mathcal{R}}
$$

The Planck analysis of CDI modes in the CMB spectrum [46] uses the variable

$$
\beta_{\mathrm{ISO}}=\frac{P_{\mathrm{CDI}}}{P_{\mathcal{R}}+P_{\mathrm{CDI}}},
$$

and for the WLI model this gives $\beta_{\text {ISO }} \sim 10^{-5}$ when $n_{s} \simeq 0.96-0.97$, which, as argued above, should also give the generic magnitude of the effect. This is still well below the state-of-theart constraints set by the Planck collaboration, yielding $\beta_{\text {ISO }} \lesssim 10^{-2}$ for generic CDI models and using different data sets [46]. Particular models of CDI modes can be constrained by an additional order of magnitude, but the predictions of our baryogenesis mechanism are still compatible with the Planck results and may be tested in the future with increased precision measurements.

The proportionality between $S_{B}$ and $\mathcal{R}$ shows that these quantities are naturally correlated, since both adiabatic and isocurvature modes are generated by thermal inflaton fluctuations. Their correlation is, however, scale-dependent, since the baryon isocurvature spectral index differs from the adiabatic spectral index,

$$
1-n_{I} \simeq \frac{d \ln P_{\mathrm{CDI}}}{d N_{e}}=\frac{d \ln P_{\mathcal{R}}}{d N_{e}}-\frac{2 n_{s}^{\prime}}{1-n_{s}} \simeq\left(1-n_{s}\right)\left[1-\frac{2 n_{s}^{\prime}}{\left(1-n_{s}\right)^{2}}\right] .
$$

The correction due to the running of the adiabatic spectral index can be significant, since in most models $n_{s}^{\prime} \sim \mathcal{O}\left(\left(1-n_{s}\right)^{2}\right)$. For instance, for thermal inflaton fluctuations and weak dissipation at horizon-crossing, $Q_{*} \ll 1$,

$$
n_{s}^{\prime} \simeq \frac{2}{3}\left(-2 \xi_{\phi_{*}}^{2}+16 \epsilon_{\phi_{*}} \eta_{\phi_{*}}-24 \epsilon_{\phi_{*}}^{2}\right),
$$

where $\xi_{\phi}^{2}=M_{P}^{4} V^{\prime \prime \prime} V^{\prime} / V^{2}$. If we consider a quartic chaotic inflaton potential, $V(\phi)=\lambda \phi^{4}$, which yields predictions for both $n_{s}$ and the tensor-to-scalar ratio $r$ in excellent agreement with the Planck results within the WLI scenario for warm inflation [19], one finds $n_{s}^{\prime}=-\left(1-n_{s}\right)^{2} / 2$, such that $\left(1-n_{I}\right) \simeq 2\left(1-n_{s}\right)$, yielding $n_{I} \sim 0.92-0.94$ for $n_{s}=0.96-0.97$. This implies, in particular, that the Planck constraints for CDI modes fully correlated with the main adiabatic component with $n_{I}=n_{s}$ do not apply in the present scenario. 
Note that for larger values of $\gamma_{X}$, the baryon-to-entropy ratio becomes less dependent on the latter, thus suppressing the associated baryon isocurvature modes. Also, when $X$ is non-relativistic, $n_{B} / s$ exhibits an additional dependence on the ratio $m_{X} / T$ that must be taken into account. This may potentially enhance $S_{B}$, depending on the particular model of warm inflation considered, although relative fluctuations in the ratio above are also typically proportional to combinations of slow-roll parameters, and hence necessarily $\mathcal{O}\left(n_{s}-1\right)$.

\section{Summary and future prospects}

In this work we have shown that particle number densities during a period of warm inflation can follow out-of-equilibrium adiabatic solutions to the Boltzmann equation and which are suppressed relative to the equilibrium value by a factor $\gamma_{X} /\left(3+\gamma_{X}\right)$, where $\gamma_{X}=\bar{\Gamma}_{X} / H$ is the ratio between the thermally averaged decay rate of the particle species $X$ of interest and the inflationary Hubble rate, obtaining a similar result for the case where scattering processes yield the dominant interactions. We have also shown numerically that the corresponding phase space-distributions tend to a stationary configuration with a modified equilibrium distribution, given by eq. (2.18), with essentially the above amplitude suppression (up to some distortion due to the momentum-dependence of the decay width) and a slightly smaller effective temperature. Such adiabatic solutions are achieved after a small number of e-folds that naturally decreases when $\gamma_{X}$ increases.

This shows that particles can remain out-of-equilibrium throughout warm inflation, with small but not necessarily negligible number densities. To illustrate the impact of this result, we have shown that the observed cosmological baryon asymmetry could be produced by the out-of-equilibrium decay of a generic $X$ particle interacting with the inflationary thermal bath, violating baryon number and $\mathrm{C} / \mathrm{CP}$. The smallness of the resulting baryon asymmetry can in this case be a consequence of the small value of $\gamma_{X}$ in combination with a small amount of $\mathrm{CP}$ violation, and also of the Boltzmann suppression in the case of non-relativistic particles. An interesting feature of this generic scenario is the generation of superhorizon baryon isocurvature modes, correlated with the main adiabatic curvature perturbations. The spectrum of such modes is model-dependent but we have shown that generically the predicted amplitude is below the current constraints on (effective) cold dark matter isocurvature perturbations by the Planck collaboration. Evidence for such modes could, in the future, constitute a smoking gun for the production of a baryon asymmetry during inflation and, in fact, for a warm inflation scenario, and the detailed properties of the spectrum may help to distinguish the present scenario from the warm baryogenesis mechanism, where a baryon asymmetry is produced directly by the dissipative effects that sustain the thermal bath during inflation.

As for the warm baryogenesis mechanism, the present scenario may also be generalized to an asymmetry in other particles carrying different charges, possibly producing an asymmetry in the dark matter sector. The resulting CDI power spectrum has an amplitude larger than the baryonic modes by a factor $\left(\Omega_{c} / \Omega_{B}\right)^{2} \sim 30$ [47], which may thus be more easily probed. 
Our results may have a significant impact in other aspects of the inflationary dynamics. For instance, it is often assumed that the particles interacting directly with the inflaton and dissipating its energy are in equilibrium with the overall thermal bath. This is required for consistently using equilibrium phase-space distribution functions to compute the associated dissipation coefficients (see, e.g., refs. [23, 25]). Typically this requires such particles to decay faster than the Hubble rate, posing constraints on the coupling constants and particle masses considered that could be relaxed if out-of-equilibrium distribution functions are known. Our results can thus potentially be employed to this effect, a possibility that we plan to investigate in detail in future work.

Another important aspect of warm inflation where the results obtained in this work should be of relevance is the primordial spectrum of curvature perturbations, since the latter depends on the phase space distribution of inflaton fluctuations. In particular, for weak dissipation at horizon-crossing, predictions for $n_{s}$ and $r$ differ significantly for the limiting cases where inflaton fluctuations are in a vacuum or in a thermal state (i.e., in equilibrium with the overall thermal bath) $[17,19]$. Although for strong dissipation this issue becomes less relevant, since dissipation becomes the dominant source of inflaton fluctuations, agreement with observations in most scenarios considered so far typically favours the $Q_{*} \lesssim 1$ regime $[17,19,48-50]$. The dissipative dynamics itself is not sufficient to determine the state of inflaton fluctuations, since other processes in the thermal bath can be responsible for a substantial creation and annihilation of inflaton particles. Since the inflaton's direct interactions with other particles cannot typically be very strong, it is unlikely that full thermal equilibrium of inflaton particles is achieved in general. Nevertheless, production of inflaton particles may play a substantial role. The adiabatic solutions obtained in this work could then be used to infer the inflaton phase-space distribution at horizon-crossing in different models, eliminating the uncertainty in observational predictions.

Adiabatic solutions to the Boltzmann equation require both the ratio $\Gamma_{X} / H$ and the equilibrium distribution $n_{X}^{\mathrm{eq}}$ to vary slowly compared to the expansion rate, thus requiring both $H$ and $T$ to remain nearly constant. This naturally makes warm inflation the type of dynamics to which such solutions can be applied.

We may envisage, however, other cosmological scenarios where, in addition to the early inflationary period in which the observable CMB scales became superhorizon, there are other (shorter) periods of inflation where particle production can sustain the temperature of the cosmic thermal bath. This may be, for instance, the case of second order or crossover cosmological phase transitions, where a scalar field rolls to a new minimum once the temperature drops below a critical value. It has been shown in ref. [51] that dissipative friction can make the field's vacuum energy dominate over the radiation energy density, and in fact prevent the latter from redshifting due to expansion. This may e.g. dilute unwanted thermal relics produced during or after the first period of warm inflation. Our solutions may, thus, also describe the evolution of the number density of different particle species during such periods, which may have a significant impact on their present abundances. One can consequently also envisage baryogenesis scenarios along the lines proposed above during these shorter inflationary periods, although these may not easily be tested if the associated isocurvature perturbations are generated at too small scales. 
We should not exclude other applications of our solutions where both particle production and an expanding environment are involved and have analogous adiabatic conditions as the ones we considered here, e.g., possibly in the quark-gluon plasma formation and subsequent hadronization process under study with heavy-ion collisions experiments.

In summary, the novel adiabatic solutions to the Boltzmann equation found in this work can have a significant impact in cosmology and shed a new light on several of its presently open questions.

\section{Acknowledgments}

A.B. is supported by STFC. M.B.-G. is partially supported by "Junta de Andalucía" (FQM101) and MINECO (Grant No. FIS1016-78198-P). R.O.R. is partially supported by Conselho Nacional de Desenvolvimento Científico e Tecnológico - CNPq (Grant No. 303377/ 2013-5) and Fundação Carlos Chagas Filho de Amparo à Pesquisa do Estado do Rio de Janeiro - FAPERJ (Grant No. E-26/201.424/2014). J.G.R. is supported by the FCT Investigator Grant No. IF/01597/2015 and partially by the H2020-MSCA-RISE-2015 Grant No. StronGrHEP-690904 and by the CIDMA Project No. UID/MAT/04106/2013.

\section{A Boltzmann equation for scattering processes}

Let us consider, for concreteness, the case where $X$ bosons can annihilate into $Y$ bosons in the thermal bath, $X X \leftrightarrow Y Y$, although our analysis can be easily generalized to different types of particles and processes such as $X Y \leftrightarrow X Y$. Labeling the $X$ particles as $(1,2)$ and the $Y$ particles as $(3,4)$, the collision term in the Boltzmann equation for the $X$ number density, eq. (2.2) is given by

$$
\begin{aligned}
C= & -\int d \Pi_{1} d \Pi_{2} d \Pi_{3} d \Pi_{4}(2 \pi)^{4} \delta^{4}\left(p_{1}+p_{2}-p_{3}-p_{4}\right)|\mathcal{M}|^{2} \\
& \times\left[f_{1} f_{2}\left(1+f_{3}^{\mathrm{eq}}\right)\left(1+f_{4}^{\mathrm{eq}}\right)-f_{3}^{\mathrm{eq}} f_{4}^{\mathrm{eq}}\left(1+f_{1}+f_{2}\right)\right]
\end{aligned}
$$

where $\mathcal{M}$ is the scattering matrix element, and we take the $Y$ particles as part of the thermal bath. Using conservation of energy and the form of the equilibrium distributions, we can show that:

$$
\frac{1+f_{3}^{\mathrm{eq}}+f_{4}^{\mathrm{eq}}}{f_{3}^{\mathrm{eq}} f_{4}^{\mathrm{eq}}}=\frac{1+f_{1}^{\mathrm{eq}}+f_{2}^{\mathrm{eq}}}{f_{1}^{\mathrm{eq}} f_{2}^{\mathrm{eq}}}
$$

such that we may write the term in square brackets in eq. (A.1), after some algebra, as

$$
\frac{f_{3} f_{4}}{f_{1}^{\mathrm{eq}} f_{2}^{\mathrm{eq}}}\left[f_{1} f_{2}-f_{1}^{\mathrm{eq}} f_{2}^{\mathrm{eq}}+\sum_{i \neq j=1,2} f_{i} f_{i}^{\mathrm{eq}}\left(f_{j}-f_{j}^{\mathrm{eq}}\right)\right]
$$

which clearly vanishes in equilibrium. The collision term can then be written in the form

$$
C=-\int \frac{d^{3} p_{1}}{(2 \pi)^{3}} \frac{d^{3} p_{2}}{(2 \pi)^{3}}\left[f_{1} f_{2}-f_{1}^{\mathrm{eq}} f_{2}^{\mathrm{eq}}+\sum_{i \neq j=1,2} f_{i} f_{i}^{\mathrm{eq}}\left(f_{j}-f_{j}^{\mathrm{eq}}\right)\right] \sigma v,
$$


where the cross section times velocity factor is given by

$$
\sigma v=\frac{\left(f_{1}^{\text {eq }} f_{2}^{\text {eq }}\right)^{-1}}{4 E_{1} E_{2}} \int d \Pi_{3} d \Pi_{4}|\mathcal{M}|^{2}(2 \pi)^{4} \delta^{4}\left(p_{1}+p_{2}-p_{3}-p_{4}\right) f_{3}^{\text {eq }} f_{4}^{\text {eq }} .
$$

The cubic terms in the phase-space distribution functions $f_{i} f_{i}^{\mathrm{eq}}\left(f_{j}-f_{j}^{\mathrm{eq}}\right)$ prevent writing the collision term in terms of the number density in a simple form, but may be discarded when $f_{i} \ll 1$, in which case $f_{i} \simeq e^{-E_{i} / T}$ (discarding chemical potentials for simplicity). In this case we can write the collision term as

$$
C \simeq-\langle\sigma v\rangle\left(n_{X}^{2}-n_{X}^{\mathrm{eq} 2}\right)
$$

where we approximated the energy dependent cross section times velocity of the scattering process by its thermal average [27], as done for the case of decays,

$$
\langle\sigma v\rangle=\frac{1}{n_{X}^{\mathrm{eq} 2}} \int \frac{d^{3} p_{1}}{(2 \pi)^{3}} \frac{d^{3} p_{2}}{(2 \pi)^{3}} \sigma v f_{1}^{\mathrm{eq}} f_{2}^{\mathrm{eq}} .
$$

This then yields eq. (2.19) for the Boltzmann equation when scattering processes dominate the collision term.

Open Access. This article is distributed under the terms of the Creative Commons Attribution License (CC-BY 4.0), which permits any use, distribution and reproduction in any medium, provided the original author(s) and source are credited.

\section{References}

[1] A.A. Starobinsky, A New Type of Isotropic Cosmological Models Without Singularity, Phys. Lett. B 91 (1980) 99 [INSPIRE].

[2] K. Sato, First Order Phase Transition of a Vacuum and Expansion of the Universe, Mon. Not. Roy. Astron. Soc. 195 (1981) 467 [INSPIRE].

[3] A.H. Guth, The Inflationary Universe: A Possible Solution to the Horizon and Flatness Problems, Phys. Rev. D 23 (1981) 347 [inSPIRE].

[4] A. Albrecht and P.J. Steinhardt, Cosmology for Grand Unified Theories with Radiatively Induced Symmetry Breaking, Phys. Rev. Lett. 48 (1982) 1220 [InSPIRE].

[5] A.D. Linde, A New Inflationary Universe Scenario: A Possible Solution of the Horizon, Flatness, Homogeneity, Isotropy and Primordial Monopole Problems, Phys. Lett. B 108 (1982) 389 [INSPIRE].

[6] A. Berera and L.-Z. Fang, Thermally induced density perturbations in the inflation era, Phys. Rev. Lett. 74 (1995) 1912 [astro-ph/9501024] [INSPIRE].

[7] A. Berera, Warm inflation, Phys. Rev. Lett. 75 (1995) 3218 [astro-ph/9509049] [InSPIRE].

[8] A. Berera, I.G. Moss and R.O. Ramos, Warm Inflation and its Microphysical Basis, Rept. Prog. Phys. 72 (2009) 026901 [arXiv: 0808.1855] [InSPIRE].

[9] M. Bastero-Gil and A. Berera, Warm inflation model building, Int. J. Mod. Phys. A 24 (2009) 2207 [arXiv: 0902.0521] [INSPIRE]. 
[10] A. Berera, Interpolating the stage of exponential expansion in the early universe: A possible alternative with no reheating, Phys. Rev. D 55 (1997) 3346 [hep-ph/9612239] [INSPIRE].

[11] A. Berera, Warm inflation at arbitrary adiabaticity: A Model, an existence proof for inflationary dynamics in quantum field theory, Nucl. Phys. B 585 (2000) 666 [hep-ph/9904409] [INSPIRE].

[12] L.M.H. Hall, I.G. Moss and A. Berera, Scalar perturbation spectra from warm inflation, Phys. Rev. D 69 (2004) 083525 [astro-ph/0305015] [INSPIRE].

[13] I.G. Moss and C. Xiong, Non-Gaussianity in fluctuations from warm inflation, JCAP 04 (2007) 007 [astro-ph/0701302] [INSPIRE].

[14] C. Graham and I.G. Moss, Density fluctuations from warm inflation, JCAP 07 (2009) 013 [arXiv:0905.3500] [INSPIRE].

[15] R.O. Ramos and L.A. da Silva, Power spectrum for inflation models with quantum and thermal noises, JCAP 03 (2013) 032 [arXiv:1302.3544] [INSPIRE].

[16] S. Bartrum, A. Berera and J.G. Rosa, Warming up for Planck, JCAP 06 (2013) 025 [arXiv:1303.3508] [INSPIRE].

[17] S. Bartrum, M. Bastero-Gil, A. Berera, R. Cerezo, R.O. Ramos and J.G. Rosa, The importance of being warm (during inflation), Phys. Lett. B 732 (2014) 116 [arXiv: 1307.5868] [INSPIRE].

[18] M. Bastero-Gil, A. Berera, I.G. Moss and R.O. Ramos, Theory of non-Gaussianity in warm inflation, JCAP 12 (2014) 008 [arXiv: 1408.4391] [INSPIRE].

[19] M. Bastero-Gil, A. Berera, R.O. Ramos and J.G. Rosa, Warm Little Inflaton, Phys. Rev. Lett. 117 (2016) 151301 [arXiv: 1604.08838] [INSPIRE].

[20] A. Berera, M. Gleiser and R.O. Ramos, A First principles warm inflation model that solves the cosmological horizon/flatness problems, Phys. Rev. Lett. 83 (1999) 264 [hep-ph/9809583] [INSPIRE].

[21] A. Berera and R.O. Ramos, Construction of a robust warm inflation mechanism, Phys. Lett. B 567 (2003) 294 [hep-ph/0210301] [INSPIRE].

[22] I.G. Moss and C. Xiong, Dissipation coefficients for supersymmetric inflatonary models, hep-ph/0603266 [INSPIRE].

[23] M. Bastero-Gil, A. Berera and R.O. Ramos, Dissipation coefficients from scalar and fermion quantum field interactions, JCAP 09 (2011) 033 [arXiv: 1008.1929] [INSPIRE].

[24] M. Bastero-Gil, A. Berera and J.G. Rosa, Warming up brane-antibrane inflation, Phys. Rev. D 84 (2011) 103503 [arXiv:1103.5623] [INSPIRE].

[25] M. Bastero-Gil, A. Berera, R.O. Ramos and J.G. Rosa, General dissipation coefficient in low-temperature warm inflation, JCAP 01 (2013) 016 [arXiv:1207.0445] [INSPIRE].

[26] R. Cerezo and J.G. Rosa, Warm Inflection, JHEP 01 (2013) 024 [arXiv:1210.7975] [INSPIRE].

[27] E.W. Kolb and M.S. Turner, The Early Universe, Front. Phys. 69 (1990) 1 [InSPIRE].

[28] D. Lyth and A. Liddle, The Primordial Density Perturbation: Cosmology, Inflation and the Origin of Structure, Cambridge University Press, Cambridge (2009). 
[29] D. Bazow, G.S. Denicol, U. Heinz, M. Martinez and J. Noronha, Analytic solution of the Boltzmann equation in an expanding system, Phys. Rev. Lett. 116 (2016) 022301 [arXiv: 1507.07834] [INSPIRE].

[30] D. Bazow, G.S. Denicol, U. Heinz, M. Martinez and J. Noronha, Nonlinear dynamics from the relativistic Boltzmann equation in the Friedmann-Lemaitre-Robertson-Walker spacetime, Phys. Rev. D 94 (2016) 125006 [arXiv:1607.05245] [INSPIRE].

[31] J.M. Cline, Baryogenesis, hep-ph/0609145 [INSPIRE].

[32] A.D. Sakharov, Violation of CP Invariance, c Asymmetry and Baryon Asymmetry of the Universe, Pisma Zh. Eksp. Teor. Fiz. 5 (1967) 32 [inSPIRE].

[33] F.R. Klinkhamer and N.S. Manton, A Saddle Point Solution in the Weinberg-Salam Theory, Phys. Rev. D 30 (1984) 2212 [inSPIRE].

[34] M. Fukugita and T. Yanagida, Baryogenesis Without Grand Unification, Phys. Lett. B 174 (1986) 45 [INSPIRE].

[35] B. Fields and S. Sarkar, Big-Bang nucleosynthesis (2006 Particle Data Group mini-review), astro-ph/0601514 [INSPIRE].

[36] D.V. Nanopoulos and S. Weinberg, Mechanisms for Cosmological Baryon Production, Phys. Rev. D 20 (1979) 2484 [INSPIRE].

[37] M. Bastero-Gil, A. Berera, R.O. Ramos and J.G. Rosa, Warm baryogenesis, Phys. Lett. B 712 (2012) 425 [arXiv:1110.3971] [INSPIRE].

[38] M.S. Turner, A.G. Cohen and D.B. Kaplan, Isocurvature Baryon Number Fluctuations in an Inflationary Universe, Phys. Lett. B 216 (1989) 20 [INSPIRE].

[39] J. Yokoyama and Y. Suto, Baryon isocurvature scenario in inflationary cosmology: A particle physics model and its astrophysical implications, Astrophys. J. 379 (1991) 427 [INSPIRE].

[40] S. Mollerach, On the Primordial Origin of Isocurvature Perturbations, Phys. Lett. B 242 (1990) 158 [INSPIRE].

[41] M. Sasaki and J. Yokoyama, Initial condition for the minimal isocurvature scenario, Phys. Rev. D 44 (1991) 970 [INSPIRE].

[42] J. Yokoyama, Formation of baryon number fluctuation in supersymmetric inflationary cosmology, Astropart. Phys. 2 (1994) 291 [INSPIRE].

[43] K. Koyama and J. Soda, Baryon isocurvature perturbation in the Affleck-Dine baryogenesis, Phys. Rev. Lett. 82 (1999) 2632 [astro-ph/9810006] [INSPIRE].

[44] D.H. Lyth, C. Ungarelli and D. Wands, The Primordial density perturbation in the curvaton scenario, Phys. Rev. D 67 (2003) 023503 [astro-ph/0208055] [INSPIRE].

[45] D. Grin, D. Hanson, G.P. Holder, O. Doré and M. Kamionkowski, Baryons do trace dark matter 380,000 years after the big bang: Search for compensated isocurvature perturbations with WMAP 9-year data, Phys. Rev. D 89 (2014) 023006 [arXiv:1306.4319] [INSPIRE].

[46] Planck collaboration, P.A.R. Ade et al., Planck 2015 results. XX. Constraints on inflation, Astron. Astrophys. 594 (2016) A20 [arXiv: 1502.02114] [INSPIRE].

[47] Planck collaboration, P.A.R. Ade et al., Planck 2015 results. XIII. Cosmological parameters, Astron. Astrophys. 594 (2016) A13 [arXiv:1502.01589] [INSPIRE]. 
[48] M. Benetti and R.O. Ramos, Warm inflation dissipative effects: predictions and constraints from the Planck data, Phys. Rev. D 95 (2017) 023517 [arXiv:1610.08758] [InSPIRE].

[49] M. Bastero-Gil, S. Bhattacharya, K. Dutta and M.R. Gangopadhyay, Constraining Warm Inflation with CMB data, arXiv:1710.10008 [INSPIRE].

[50] R. Arya, A. Dasgupta, G. Goswami, J. Prasad and R. Rangarajan, Revisiting CMB constraints on Warm Inflation, arXiv:1710.11109 [INSPIRE].

[51] S. Bartrum, A. Berera and J.G. Rosa, Fluctuation-dissipation dynamics of cosmological scalar fields, Phys. Rev. D 91 (2015) 083540 [arXiv:1412.5489] [INSPIRE]. 